\title{
Research on the Nursing Modes for the Elderly with Dementia
}

\author{
Sun, Lingxue/ $\mathrm{PhD}$ candidate \\ School of Public Affairs, Zhejiang University \\ Hangzhou, China \\ sunlx@zju.edu.cn
}

\begin{abstract}
China's population structure is undergoing an important period of rapid aging and development. The number of dementia patients has been increasing since recent years. As a result, it is significant to take good care of the elderly with dementia and ease the burden of their family and the society. This paper analyzes different nursing modes, including the professional organization care mode, the home care mode, the day care center mode, and the community mutual care mode. Then it builds a mode of community mutual assistance that combines "a professional integrated day care center with old-age support points based on household assistance activities". The community mutual mode mainly achieves two goals, namely the availability of services and cost savings. Finally, this paper puts forward four suggestions, which includes doing a good job in the construction of supporting infrastructure, improving the social support network for dementia elderly people, establishing a longterm mechanism for financial investment and strengthening the long-term care insurance system.
\end{abstract}

Keywords-Elderly with dementia; Nursing modes; Long-term care; Community mutual care

\section{INTRODUCTION}

Due to the rapid development of China's economy and society as well as the intervention of the national policy on family planning, China's aging population continues to deepen under the dual effect of a prolonged life expectancy of the population and a rapidly declining fertility rate. Closely related pension services will become more and more important in the future. According to the results of the survey conducted by the Aged Committee, the number of people over the age of 60 in China has exceeded 210 million in 2015, among which dementia elderly account for about 8 Million. This disease occurs mainly in the elderly population over 60 years of old, and it is manifested by the constant deterioration of cognitive and memory functions. In the middle and late stages of dementia patients, it loses basic daily life ability and is accompanied by the corresponding disability.

The caregivers of the elderly with dementia not only has to bear the heavy physical labor, but also has to deal with some weird behavioral consequences of the patients. At the same time, the long illness period also leads to high medical and nursing costs. Some of the caregivers lack professional nursing knowledge, and the disposable time of the caregiver is not compatible with the long-term caregiving needs of the demented elderly themselves. As a result, it not only makes the condition of the elderly worsen, but also brings the economic and spiritual burdens on family members. In China's current pension policy, there is no specific policy formulation and institutional arrangement for the demented elderly, and related institutions lack professional nursing personnel. So how to effectively mobilize the family, society, and government to establish a cost-effective and demented care model for the elderly will be significant for our society.

\section{DEFINITION AND PROBLEMS OF DEMENTIA PATIENTS}

Dementia is a condition in which the brain is degraded and has at least one cognitive deficit due to a progressive, irreversible functional deterioration. The typical process last approximately 8-12 years (Agronin, 2008) [1]. According to relevant predictions, the incidence of dementia in China will be five times by 2050 compared to current situation (Lily Dongxia Xiao, et al., 2014) [2]. Dementia is a chronic disease that costs quite a lot. As the country with the fastest growing population of the elderly, the time and money cost of Alzheimer's patients is a huge challenge for China (Joaqui' $\mathrm{n}$ F Mould-Quevedo, et al., 2013) [3]. The study found that people who take care of the demented elderly suffer more pressure than ordinary informal caregivers, and they are more likely to feel isolated from the society and also more prone to meet chronic health problems and depression (Rabins et al. 1982) [4].

Therefore, the status quo and difficulties of dementia care for the elderly are mainly reflected in three aspects: First, the number of elderly people with mental retardation increases year by year, and the possible occurrence of post-morbidity are further increased. Moreover, the corresponding medical and nursing costs increased quickly. The second is that the dementia elderly care resources are in short supply. The traditional family caregivers need to sacrifice their own health and welfare to take care of the elderly. In addition, they lack of professional knowledge which also prevents the elderly from receiving early rehabilitation and prevention. Third, there is a lack of relevant policies and systems for the elderly with dementia. Compared to some developed countries, China has not yet established relevant policies specifically for demented elderly people, and it lacks a complete social support network system to provide appropriate services and resources more effectively. 


\section{COMPARISON OF NURSING MODES}

The modes of care for the demented elderly is usually divided into formal care and informal care, or home care, community care and institutional care according to the location of the service. Moreover, according to the type of service, it can be divided into person-centered care, dementia-care mapping, and usual care (Lynn Chenoweth, 2009) [5].

\section{A. Professional organization care mode}

The concept of care for group homes originated in Sweden and was introduced in Japan in 2000. The group houses emphasize the use of community, and the atmosphere tends to be warm. This mode takes 24 hours of care, and lets the demented old people feel like returning home. In recent years, care models have also emerged in the United States for assisted care facilities, and patients' spouses can stay with them. In fact, this type of organization centralizes allocation of nursing services and facility resources, which enable dementia elderly people to enjoy convenient and timely care while living freely.

At present, in respect of institutional care for demented elderly people, the emphasis is placed on the physical and mental feelings of the elderly. Without interfering with the normal life of the elderly, the organization can provide necessary assistance that elderly cannot finish by themselves. For the majority of developing countries with low income elderly people groups, they cannot afford high institutional care costs. They should consider the availability of services that are more accessible and cost-effective.

\section{B. Home care mode}

In Sweden and Japan, there is also an efficient care model called "Omsorg care", which meets the needs of people who receive care and also supports self-supporting work. These caregivers study basic medical knowledge and are equivalent to the occupations that combine nurses, home attendants, and caregivers. In Sweden, the majority of demented older people who use Under Nurse's help are kept at a mild level and live in their own homes. They only receive an average of 15 minutes of home-based services each day, and $45 \%$ of them are living alone. The special orientation of functions on caregiving strengthens the professional of the work and can also attract more people to participate in it.

In contrast, due to the lack of professionals in our country, it is unrealistic to implement professional service on a daily basis in a short period of time. The better way is to find a relatively fixed place, with 1 to 2 professionals. Caregivers and retired seniors in the community are conduct training and assignments to provide guidance and support for informal care, and professionals take into consideration the needs of community day care centers.

\section{Day care center mode}

There are two typical day care centers in the Netherlands, and they are the traditional Nursing Home-based (NH) and the Meeting Centers Support Programme (MCSP). The NH model aims to provide temporary day care for dementia patients who are waiting to enter nursing homes (de Jong and Boersma, 2009) [6]. Moreover, around the 1980s, a more socialized and integrated community support program designed for mild to moderate dementia patients and their families developed in the Netherlands (Dröes et al., 2006) [7] . The use of community activity centers enables people with dementia and their caregivers to access the places more easily, and in a more timely and targeted manner to help people with dementia and their caregivers to adapt to the impact of dementia on life (Dröes et al., 2004) [8]. In addition, Netherlands also pioneered the dementia intervention activation center. It is characterized by the provision of a daycare center to provide intervention and activation training for patients with dementia in the early stages, in order to help them to establish a variety of conditional reflexes and learning abilities. Therefore, in this type of mode is built with relatively small staffing, low labor costs, and relatively low hardware requirements.

\section{Community mutual care mode}

In recent years, Germany has embarked on a model of mutual support for the elderly called "Old Home." Some elderly people who are afraid of loneliness and are unwilling to go to nursing homes voluntarily organize their own small world. In the "Old Home," members share the housework, help each other, and participate in social activities. In these communities, not only older people can join in but also young people can join. Participants can choose hourly wages, or they can deposit service hours into the cooperative to get free service in the future.

In Taiwan, there is a form of mutual care for the dementia elderly. The community provides more care during the day time. A community worker is responsible for coordinating the work and the care of the demented elderly is jointly completed by the family members. In addition, Qingdao has created a unique family mutual support mode. The elderly live in the vicinity to enjoy entertainment and leisure during the day to the mutual aid point. Qingdao government provides monthly operating subsidies for each mutual aid point with $100 \mathrm{RMB}$, which effectively solve the lack of old-age service places problems. 


\section{ADDRESS SELECTION FOR THE DEMENTIA ELDERLY} CARE

This article will choose the form of a combination of community mutual assistance stations and mutual support for the elderly. The community mutual aid station is only equipped with 1-2 professional staff, and they are mainly responsible for the day care. In the mutual aid and old-age care centers, the main tasks are home-based housework assistance and daily contact for demented elderly people, and volunteers in the community are responsible for the main job.

First of all, community mutual aid stations mainly provide day care places for demented elderly people. It also promotes mutual care station workers, family members and community mutual help volunteers to coordinate the care of demented elderly people. In order to keep abreast of the whereabouts of demented elderly people and prevent them from losing, it is also very important to establish a mutual contact point for the community. The main function of the community contact point is to contact the designated contact person promptly when the elderly person is found to be traveling alone, and to inform the family care worker of the elderly in time.

According to the linear programming method and singlelayered location model used in the process of constructing a community health service center location model in conjunction with Song Jie et al. (2009) and Zhang Afen (2010) [9][10], this paper focuses on the central site in the community mutual aid service network. Community mutual service stations establish corresponding site selection models. For the site selection study, the goal is to ensure that residents have the shortest distance from the corresponding service sites. The objective function studied in this paper is also to ensure that all residential sites in the planning area have the shortest total distance from the corresponding depersonalized elderly community mutual service stations.

Before establishing the model, first we make the following assumptions: 1. Settlement treatment for residential areas to facilitate calculation; 2 . Determine the service radius, first we ensure that the "15-minute" health circle. According to the average person walking speed $5 \mathrm{~km} / \mathrm{h}$, we convert it to the distance calculation, that is, the distance to the 15-minute health circle is 1.25 kilometers. Considering the population density in the planning area and the number of demented elderly people, the service radius is adjusted according to the actual situation in the model calculation process. 3 . The communities mutual aid stations provide homogenous services; 4. The construction of community mutual help service stations belongs to public medical infrastructure construction, so the related costs such as construction costs are not considered.

After determining the basic goals of the location model, we establish the following model:

$$
\begin{aligned}
& \operatorname{Min} \sum_{(i \in I)} \sum_{(j \in J)} h_{i} d_{i j} x_{i j} \\
& \text { s.t } \sum_{(i \in I)} x_{i j}=1 \quad \forall i \in I, \quad j \in J \\
& \sum_{(j \in I)} y_{j}=P \quad \forall j \in J \\
& x_{i j}-y_{j} \leq 0 \quad \forall i \in I, \quad j \in J
\end{aligned}
$$

$$
\begin{gathered}
\mathrm{d}_{\mathrm{ij}} \leq \mathrm{R} \\
\mathrm{x}_{\mathrm{ij}} \in\{0,1\} \quad \forall \mathrm{i} \in \mathrm{I}, \quad \mathrm{j} \in \mathrm{J} \\
\mathrm{y}_{\mathrm{j}} \in\{0,1\} \quad \forall \mathrm{j} \in \mathrm{J}
\end{gathered}
$$

$\mathrm{h}_{\mathrm{i}}$ is the number of demented elderly people in residential area $i$, that is the demand for care;

$\mathrm{d}_{\mathrm{ij}}$ is the distance from the candidate point $\mathrm{i}$ to $\mathrm{j}$ candidate;

$\mathrm{P}$ is the number of new community mutual service stations;

$\mathrm{x}_{\mathrm{ij}}$ is whether the residential point $\mathrm{i}$ can be covered by a certain service point. If it is covered, then $\mathrm{x}_{\mathrm{ij}}=1$; If it is not covered by any service point, then $\mathrm{x}_{\mathrm{ij}}=0$;

$y_{j}$ is whether to set the service point at point $\mathrm{j}$. If set, $\mathrm{y}_{\mathrm{j}}=1$, if not, then $\mathrm{y}_{\mathrm{j}}=0$.

Among them, the objective function (1) is to minimize the distance between demented old people and community mutual service stations when the demand is met. Constraints (2) ensure that each demand point can be covered by only one service point. Constraints (3) is the number of community mutual aid service stations, which does not exceed the maximum number of total resource constraints, and can be appropriately increased or decreased according to population density. Constraints (4) ensure that demand points can only be covered by the selected service points. And constraints (5) ensure that all residential areas are at a distance of less than $\mathrm{R}$ from the community mutual aid service stations, where the initial service radius is based on the "15-minute health circle" and appropriate adjustments are made based on actual population density needs. Constraints (6) and (7) satisfy the variable definition.

\section{CONCLUSION}

This article starts with home and community endowment, and builds a model of community mutual assistance that combines a "professional integrated day care center with oldage support points based on household assistance activities". The community mutual mode mainly achieves two goals. First, it provides the availability of services. It not only includes regular dementia elderly activation interventions, psychological counseling and other professional services, but also supply timely assistance on the care of daily living. Second, it realizes cost savings. Through the combination of home and community endowment methods, this mode can not only improve the quality of life of demented people while reducing the economic burden of the elderly, but also maximize the human resources of the community by fully mobilizing the community's human resources. It also save labor costs and operating costs.

Due to the existing problems, the paper puts forward the following suggestions: First, do a good job in the construction of supporting infrastructure. Based on the construction of community mutual care model proposed in this paper, it is necessary to implement the distribution plan of community mutual aid sites. At the same time, it needs to determine the corresponding number of sites and service radius according to the scale of demented elderly people and the density of demented elderly population in the planning area. Second, we 
should improve the social support network for dementia elderly people. It means to encourage community volunteers to participate in old-age care, ease the care burden of family caregivers, and establish a good social relationship. Meanwhile, it is still necessary to emphasize the family member's responsibility for care and to encourage family members to participate as much as possible as primary members in the care activities. The third one is to establish a long-term mechanism for financial investment. Emphasis on the role of the government will provide a certain degree of assistance to lowincome and demented people in a vulnerable position to help them have enough money to pay the care costs. The fourth one is to strengthen the long-term care insurance system. China's long-term care insurance system is still in the exploration stage. In the process of establishing and improving the long-term care insurance system, the elderly with dementia should be included in the system. Therefore, it provides long-term care protection for the demented elderly from the social perspective.

\section{REFERENCES}

[1] Agronin, M. E. (2008). Alzheimer Disease and other dementias: A practical guide. Philadelphia, PA: Wolters Kluwer Health/Lippincott Williams \& Wilkins.

[2] Lily Dongxia Xiao, Jing Wang, Guo-Ping He, Anita De Bellis, Jenny Verbeeck and Helena Kyriazopoulos. Family caregiver challenges in dementia care in Australia and China: a critical perspective. BMC Geriatrics (2014), 14:6.

[3] Joaquı 'n F Mould-Quevedo*, Boxiong Tang, Eran Harary, Ricky Kurzman, Sharon Pan, Jiyue Yang and Juan Qiao. The burden of caring for dementia patients: caregiver reports from a cross-sectional hospitalbased study in China. Expert Rev. Pharmacoecon. Outcomes Res. 13(5), 663-673 (2013).

[4] Rabins P.V., Mace N.L. \& Lucas M.J. The impact of dementia on the family. Journal of the American Medical Association (1982) 248:333335.

[5] Lynn Chenoweth, Madeleine T King, Yun-Hee Jeon, Henry Brodaty, Jane Stein-Parbury, Richard Norman, Marion Haas, Georgina Luscombe.Caring for Aged Dementia Care Resident Study (CADRES) of person-centred care, dementia-care mapping, and usual care in dementia: a cluster-randomised trial. Lancet Neurol (2009); 8: 317-25.

[6] De Jong, J. D. and Boersma, F. Dutch psychogeriatric day-care centers: a qualitative study of the needs and wishes of carers. International $\begin{array}{llll}\text { Psychogeriatrics } & \text { 2609), 268-277. }\end{array}$ $10.1017 / \mathrm{S} 1041610208008247$.

[7] Dröes, R. M., Meiland, F. J. M., Schmitz, M. J. and van Tilburg, W. Effect of the meeting centres support program on informal carers of people with dementia: results from a multi-centre study. Aging Mental Health (2006), 10, 112-124. DOI: 10.1080/13607860500310682.

[8] Dröes, R. M., Breebaart, E., Meiland, F. J. M., van Tilburg, W. and Mellenbergh, G. J. Effect of meeting centres support program on feelings of competence of family carers and delay of institutionalization of people with dementia. Aging Mental Health (2004), 8, 201-211. DOI: 10.1080/13607860410001669732.

[9] Song Jie, Li Binfeng, Yang Wei, Tao Hui, Wu Su.Study on Location Selection of Urban Community Health Service Based on Linear Programming [J]. Chinese Health Resources, 2009, (02):86-88. (In Chinese)

[10] Zhang Afen. Study on the Location of Community Health Service Organizations [D]. Huazhong University of Science and Technology, 2010. (In Chinese) 